\title{
Grating Lobe Reduction in Transducer Arrays Through Structural Filtering of Supercritical Plates
}

Brian E. Anderson

Stephen A. Hambric

Jack W. Hughes

Follow this and additional works at: https://scholarsarchive.byu.edu/facpub

Part of the Astrophysics and Astronomy Commons, and the Physics Commons

\section{Original Publication Citation}

B. E. Anderson, W. J. Hughes, and S. A. Hambric, "Grating lobe reduction in transducer arrays through structural filtering of supercritical plates," J. Acoust. Soc. Am. 126, 612-619 (29).

\section{BYU ScholarsArchive Citation}

Anderson, Brian E.; Hambric, Stephen A.; and Hughes, Jack W., "Grating Lobe Reduction in Transducer Arrays Through Structural Filtering of Supercritical Plates" (2007). Faculty Publications. 856.

https://scholarsarchive.byu.edu/facpub/856 accepted for inclusion in Faculty Publications by an authorized administrator of BYU ScholarsArchive. For more information, please contact ellen_amatangelo@byu.edu. 


\title{
Grating lobe reduction in transducer arrays through structural filtering of supercritical plates
}

\author{
Brian E. Anderson, ${ }^{\text {a) }}$ W. Jack Hughes, and Stephen A. Hambric \\ Applied Research Laboratory, The Pennsylvania State University, P.O. Box 30, State College, \\ Pennsylvania 16804
}

(Received 24 October 2008; revised 3 June 2009; accepted 4 June 2009)

\begin{abstract}
The effect of placing a structural acoustic filter between water and the transducer elements of an array to help reduce undesirable grating lobes is investigated. A supercritical plate is mounted to transducer elements with a thin decoupling polyurethane layer between the transducers and the plate. The plate acts as a radiation/incidence angle filter to pass energy at angles near normal incidence, but suppress energy at large incidence angles. Grating lobe reduction is achieved at the expense of limiting the available steering of the main lobe. Within this steer angle limitation, the main lobe can be steered as normal while the grating lobe level is reduced by the plate's angular filtering. The insertion of a plate structural filter provides, in principal, an inexpensive and easily implemented approach to extend usable frequency bandwidth with reduced level grating lobes, without increasing the number of array elements. Even though the data match theory well, a practical material has yet to be found that possesses optimal material properties to make the proposed idea practical. This work represents the first attempt to advantageously utilize a plate above its critical frequency to provide angular dependent sound transmission filtering.
\end{abstract}

(C) 2009 Acoustical Society of America. [DOI: 10.1121/1.3159366]

PACS number(s): 43.38.Hz, 43.30.Yj, 43.40.Rj, 43.55.Rg [AJZ] Pages: 612-619

\section{INTRODUCTION}

According to traditional array theory, the usable frequency bandwidth of an array of regularly spaced point sources is limited according to its spacing. ${ }^{1-3}$ The optimal spacing, $d$, is typically set equal to half the desired center frequency wavelength, $\lambda$, [Fig. 1(a) displays a point source line array with these geometrical properties identified]. Thus, the resulting array may be operated below and above the center frequency, within certain limitations. Below the center frequency, the radiated beam widens and approaches an omni-directional beam pattern at very low frequencies. Above the center frequency, the radiated beam narrows. At frequencies greater than or equal to twice that of the center frequency, grating lobes appear in the radiated beam pattern as duplicates of the main beam of sound energy (i.e., duplicates in radiation level, but not in the direction radiated). These undesirable grating lobes result from an aliasing effect due to the discrete nature of an array of elements, the regular spacing of elements, and the relationship between the wavelength and inter-element spacing.

The above discussion assumes that the main energy is sent in a direction normal to the plane of the array. An array may also be used to steer sound radiation in different directions by introducing appropriate electronic phases to the array elements. When an array is steered, the grating lobe(s) appear in the beam pattern at frequencies lower than twice the center frequency. Grating lobes in radiation beam patterns cause operational confusion for technology that relies

\footnotetext{
a) Author to whom correspondence should be addressed. Present address: Department of Physics and Astronomy, Brigham Young University, N283 ESC, Provo, Utah 84602. Electronic mail: bea@byu.edu
}

on arrays, such as medical ultrasound and underwater SONAR. In transmit mode, grating lobes cause an array to transmit sound energy in unintended directions. In receive mode, grating lobes prevent an array's ability to detect the direction of incoming sound energy. However, larger frequency bandwidth allows higher resolution and greater ranging capabilities in applications where both transmit and receive modes are used.

There have been many different attempts to reduce or eliminate grating lobes in radiation patterns in SONAR array applications and in medical ultrasound array applications. SONAR arrays are often designed for high source level output in a narrow frequency band and are not traditionally used in the broad frequency range such as that used in the ultrasound community. Consequently, the majority of studies involving grating lobe reduction have traditionally come from the ultrasound community. The most commonly used techniques, aimed at grating lobe reduction in directivity patterns, attempt to break up the regular element spacing or regular element size which gives rise to grating lobes. Current techniques of reducing grating lobes include sparse element spacing, ${ }^{4-17}$ annular rings of elements, ${ }^{18-22}$ use of adjacent staggered arrays, ${ }^{23-25}$ pseudorandom continuous wave (cw) signals, ${ }^{26-28}$ and exploitation of harmonic imaging. ${ }^{29}$

There are many existing arrays with regularly spaced elements that have no practical means for reducing their grating lobes. None of the methods mentioned above are practical solutions to retrofit onto these array systems. The methods mentioned above either require changes to the original array design, require impractical modifications to the input signals, and/or are not practical solutions for underwater SONAR arrays. A technique is needed that directly eliminates grating lobes, which could be retrofitted onto existing array 
(a)

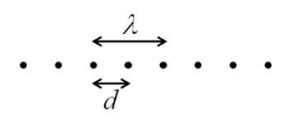

(b)

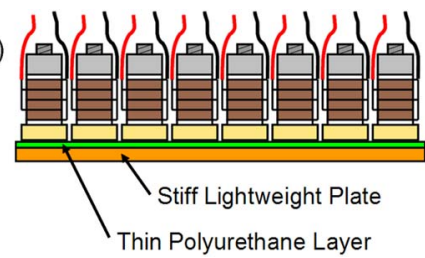

FIG. 1. (Color online) (a) Illustration of a point source line array. The variable $d$ represents the center to center spacing between adjacent transducer elements. The variable $\lambda$ represents the wavelength (in this case the center frequency wavelength used to design the array). (b) Illustration of a typical Tonpilz line array mounted onto a stiff lightweight plate. A thin layer of polyurethane was placed between the array and the plate.

designs. Grating lobe reduction would provide these existing arrays with better ranging capabilities and larger frequency bandwidth and therefore better image resolution compared to existing array capabilities. The goal of this paper is to present a new method to reduce grating lobes through a retrofitting approach.

The proposed technique for grating lobe level reduction presents a different approach to the problem than other methods. An extremely stiff and lightweight plate, operated at frequencies above its critical frequency, is placed in front of the transducer array to provide a radiation angle filter (and incidence angle filter) achieved through exploitation of the coincidence effect (a sound and structure interaction principle). ${ }^{30}$ Figure 1(b) provides a simple illustration to show a typical eight element Tonpilz transducer line array that has been bonded onto a plate (or a bar in this case). The idea behind this technique was developed as an extension of the thesis work by Hutto, ${ }^{31}$ Grosh, ${ }^{32}$ and Phillips. ${ }^{33}$

A previous paper written by the authors showed that an array may steer sound energy through a supercritical plate without refraction and without widening the main lobe of energy, meaning that the plate does not alter the intended electronic phasing required to steer an array. ${ }^{34}$ Sound energy passes through the plate at normal incidence and radiation angles less than the coincidence angle are attenuated somewhat due to the mass of the plate. However, sound energy at large radiation angles, greater than the coincidence angle, will be attenuated due to the stiffness of the plate. If the plate's material properties and geometry are selected appropriately and the array is operated within certain constraints, then grating lobe levels may be greatly reduced while main lobes are passed by the radiation angle filter. The insertion of a plate would provide an inexpensive and easily implemented approach to eliminating grating lobes without adjusting the number or positions of array elements.

This paper introduces a method to reduce grating lobes. However, the plate used for grating lobe reduction in this work does not provide a practical solution to the problem, as will be shown. While grating lobes are reduced by the plate used, additional relative increases in side lobe levels, combined with a reduction in the main lobe level, makes the plate used in this study an impractical solution to the problem. Thus this paper is intended to provide the blue print to design a passive structural acoustic filter to attenuate grating lobes when a plate material with sufficient stiffness to mass properties is developed.

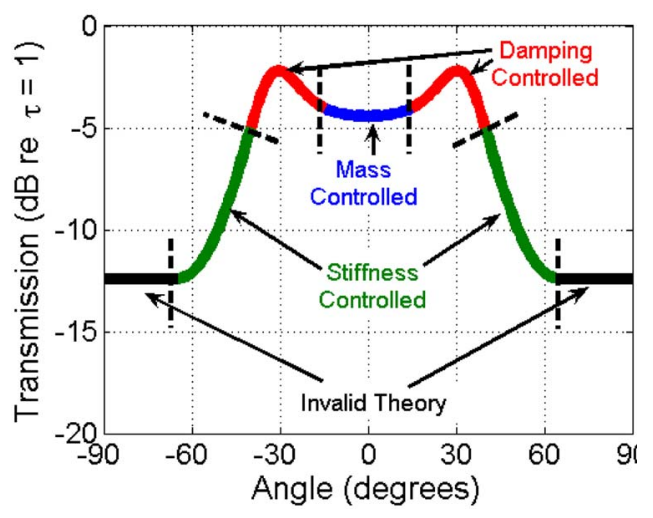

FIG. 2. (Color online) Example of the transmission through a supercritical plate which possesses a high stiffness to mass ratio at a single frequency versus angle of incidence.

\section{THEORETICAL DEVELOPMENTS}

\section{A. Angular filtering}

The critical or coincidence frequency of a plate is the frequency at which the bending wave speed in the plate equals the acoustic fluid wave speed. A high degree of sound transmission exists at grazing incidence for plates at their critical frequencies. Below this critical frequency, the sound transmission through the plate is governed by the well known mass law and is independent of incidence angle. However, above the critical frequency (the supercritical region), the transmission through the plate varies significantly with angle of incidence. Figure 2 shows an example of the transmission versus angle for a supercritical plate possessing a high stiffness to mass ratio. The mass of the plate governs the transmission at normal incidence and at angles near normal incidence. At an angle called the coincidence angle, $\theta_{\mathrm{CO}}$, the reactive part of the plate's impedance goes to zero, and the sound transmission is dominated by the amount of damping within the plate. ${ }^{30}$ Thus, instead of a high degree of sound transmission at grazing incidence for the critical frequency, the high degree of sound transmission shifts to coincidence angles in the forward $180^{\circ}$. At angles greater than the coincidence angle, the transmission is dominated by the plate's stiffness.

A derivation of an expression for the angular dependent sound transmission coefficient, $\tau$, through supercritical plates is given by Fahy and Gardonio ${ }^{30}$ in their Eq. (5.38a):

$$
\tau=\frac{(2 \rho c \sec \theta)^{2}}{\left[2 \rho c \sec \theta+\frac{1}{\omega} D \eta k^{4} \sin ^{4} \theta\right]^{2}+\left[m \omega-\frac{1}{\omega} D k^{4} \sin ^{4} \theta\right]^{2}},
$$

where $\rho$ is the density of the acoustic fluid, $c$ is the speed of sound in the acoustic fluid, $\theta$ is the angle of incidence, $\omega$ is the angular frequency, $D$ is the plate's stiffness, $\eta$ is the internal damping in the plate, $k$ is the acoustic wavenumber in the fluid, and $m$ is the mass per unit area in the plate. The transmission loss through the plate is 


$$
\mathrm{TL}=20 \log _{10} \frac{1}{\tau} .
$$

One may consider the coincidence angle to be the "cutoff angle" of the angular filter (similar to the cutoff frequency in frequency domain filtering). The range of angles between the two coincidence angles may be considered to be the angular pass band region. Finally, the angles between grazing incidence and the coincidence angles may be considered the angular stop band region. Fahy and Gardonio commented that Eq. (1) does not model the angular region $\pm 78^{\circ}- \pm 90^{\circ}$ (from normal incidence) near grazing incidence in practice $^{30}$ (at grazing incidence, the expression converges to a value of 1 , regardless of the frequency used, meaning no loss in transmission). The authors came to a similar conclusion from their evaluations of experimental data; however, the authors have decided that the expression in Eq. (1) is invalid from grazing incidence to the first null in the expression that typically occurs at $\pm 65^{\circ}$ (see Fig. 2).

To use a plate as an angular sound filter, the plate's material properties and thickness should be chosen carefully to minimize attenuation of the pass band region and maximize the attenuation in the stop band region. Figure 2 is a good example of a filter which attenuates the stop band more than it attenuates the pass band. Another way to look at this angular filtering is that it increases the directivity in the forward direction, which may be beneficially exploited in other applications.

In selecting the optimum plate thickness, one must decide what coincidence angle, or cutoff angle, would be desired. In addition, one should decide the lowest frequency at which this cutoff angle is desired, keeping in mind that as frequency decreases the coincidence angle increases (thus the pass band region widens) according to the following equation:

$$
\sin ^{2} \theta_{\mathrm{CO}}=\frac{c^{2}}{c_{B}^{2}}=\frac{c^{2}}{\omega} \sqrt{\frac{m}{D}},
$$

where $c_{B}$ is the bending wave speed for a thin plate (thin plate theory ignores the effects due to shear deformation and rotary inertia). The parameters $D$ and $m$ may be written in terms of the plate's material properties and thickness as

$$
\begin{aligned}
& D=\frac{E h^{3}}{12\left(1-\sigma^{2}\right)}, \\
& m=\rho_{P} h,
\end{aligned}
$$

where $E$ is the plate's Young's modulus of elasticity, $h$ is the plate's thickness, $\sigma$ is the plate's Poisson ratio, and $\rho_{P}$ is the volumetric density of the plate. Equations (3)-(5) are adapted from Fahy and Gardonio's Eqs. (5.42)-(5.43). If one solves Eq. (3) for $h$ and substitutes the desired cutoff angle with the selected frequency, then the interdependence of the thickness and the material properties may be examined (see Chap. 3 of the thesis by Anderson ${ }^{35}$ for further details). Solving for $h$ yields

$$
h=\frac{c^{2}}{\omega \sin ^{2} \theta_{\mathrm{CO}}} \sqrt{\frac{12 \rho_{P}\left(1-\sigma^{2}\right)}{E}} .
$$

If a specific plate material is selected, then Eq. (6) may be used to calculate the thickness required to set the target coincidence angles at the desired operation frequency. As an example, the measured material properties of an alumina bar used in this study are $E=391 \mathrm{GPa}, \rho_{P}=3956 \mathrm{~kg} \mathrm{~m}^{3}$, and $\sigma=0.22$. The desired coincidence angle was set to be $\theta_{\mathrm{CO}}$ $=30^{\circ}$ at a frequency of $f=50 \mathrm{kHz}$. Thus the optimal plate thickness for alumina in this case is $h=9.7 \mathrm{~mm}$.

If Eq. (1) is evaluated at normal incidence, then one may estimate the expected pass band loss for an undamped ( $\eta$ $=0)$ supercritical plate angular filter. The expected pass band transmission loss for an alumina plate of thickness $h$ $=9.7 \mathrm{~mm}$ is $12.3 \mathrm{~dB}$. The stop band transmission loss may be determined at the minima of Eq. (1) that are located between grazing incidence and the coincidence angles. For the alumina plate, the minima are located at $\pm 65^{\circ}$ resulting in a maximum stop band transmission loss of $24.3 \mathrm{~dB}$. Thus the supercritical plate may be used as a passive filter with a relative stop band transmission loss of $12 \mathrm{~dB}$ at large angles relative to the pass band region.

The directivity pattern, $H(\theta)$, without a plate may be calculated from typical line array theory (first term in curly brackets) multiplied by the single element directivity (second term in curly brackets), ${ }^{1-3,35}$

$$
\begin{aligned}
H(\theta)= & \left\{\frac{1}{N} \frac{\sin \left[\frac{N k d}{2}\left(\sin \theta-\sin \theta_{0}\right)\right]}{\sin \left[\frac{k d}{2}\left(\sin \theta-\sin \theta_{0}\right)\right]}\right\} \\
& \times\left\{\frac{\sin \left[\frac{1}{2} k a \sin \theta\right]}{\frac{1}{2} k a \sin \theta}\right\},
\end{aligned}
$$

where $a$ is the width of a rectangular head transducer, and $N$ is the number of array elements. The predicted angular dependent transmission coefficients [Eq. (1)] for an alumina plate were then multiplied by the without-plate directivity pattern to yield the with-plate directivity patterns. Figure 3 displays theoretical directivity patterns without and with the insertion of an alumina plate at $50 \mathrm{kHz}$. At an angle of $-45^{\circ}$ a reduction of $9 \mathrm{~dB}$ in the grating lobe level occurs due to the insertion of the plate. One may also notice the unfortunate relative increase in the transmission of side lobe levels centered at the predicted $\theta_{\mathrm{CO}}\left( \pm 30^{\circ}\right)$ due to the high degree of sound transmission at these angles. The relative level increase at the coincidence angles depends on $m$ since the depth of the pass band depends on $m$. This is one of the major issues in the use of a supercritical plate for angular filtering. The optimal plate material would have a low $m$ while maintaining a high $D / m$. The thesis by Anderson ${ }^{35}$ includes a discussion of ideal plate materials in Chaps. 3 and 7. It is important to note that the discussion given in Anderson's thesis pertains to underwater arrays with design fre- 


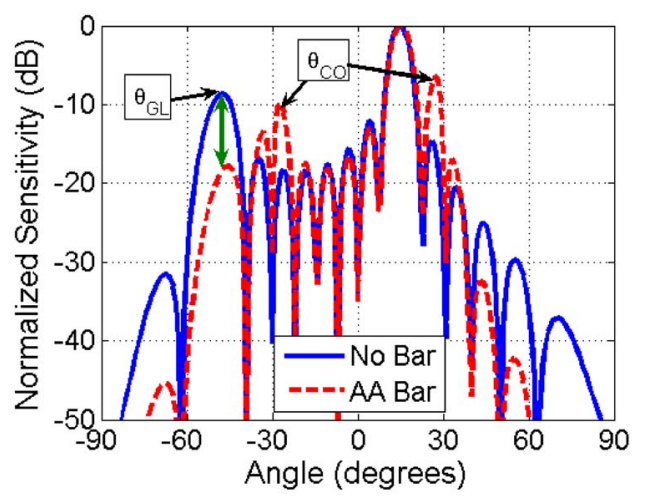

FIG. 3. (Color online) Example of grating lobe filtering through the addition of a supercritical plate. The plot displays theoretical sensitivity patterns at 50 $\mathrm{kHz}$, steered to $15^{\circ}$, obtained from a point source directivity function with the addition of assumed single element directivity.

quencies of $25 \mathrm{kHz}$ since the ideal material properties will depend on the desired frequency range.

\section{B. Frequency response}

An equivalent circuit will now be presented from which equations can be derived to model the frequency response of an array with and without the insertion of a plate. The mechanical impedance domain equivalent circuit for a plate loaded transducer in a receive condition is given in Fig. 4. A normal incidence plane wave with a first-order resistive loading approximation models the acoustic fluid medium. The incident wave impinges upon the plate, which is modeled by a waveguide T-network (the expressions used in T-networks are given by Mason on pp. 204-205 in Ref. 36). The thin layer of polyurethane between the plate and the transducer elements is also modeled by a waveguide T-network. Finally, a lumped-element equivalent circuit is given for the piezoelectric Tonpilz transducer. ${ }^{2}$ The output potential signal of the equivalent circuit is proportional to the voltage signal measured at the transducer terminals.

A transfer function, $\mathrm{TF}_{\text {with plate }}$, between the input plane pressure wave, $\hat{p}$, and the output voltage signal, $\hat{V}$, may be derived to model the measured frequency response using standard circuit analysis techniques:

$$
\begin{aligned}
& \mathrm{TF}_{\text {with plate }}=\frac{\hat{V}}{\hat{p}}=\frac{S}{\phi} \frac{Z_{P 2} Z_{C 2} Z_{0}}{Z_{\alpha} Z_{\beta} Z_{\gamma}}, \\
& Z_{\alpha}=Z_{A}+Z_{P 1}+Z_{P 2}, \\
& Z_{\beta}=Z_{P 1}+Z_{P 2}+Z_{C 1}+Z_{C 2},
\end{aligned}
$$

$$
Z_{\gamma}=Z_{C 1}+Z_{C 2}+Z_{T 1}+Z_{T 0},
$$

where $S$ is the surface area of a single transducer, $\phi$ is the electro-mechanical coupling factor, and $Z$ represents a mechanical impedance quantity. The subscripts designate the following: resistive acoustic loading $A$, plate's masslike quantity $P 1$, plate's compliancelike quantity $P 2$, compliant layer's masslike quantity $C 1$, compliant layer's compliantlike quantity $C 2$, transducer's lumped mechanical model $T 1$ (including mass, compliance, and resistance), and transducer's electrical capacitance $T 0$. The transfer function for an unloaded transducer (no plate), $\mathrm{TF}_{\text {no plate }}$, is found by setting $Z_{P 1}=Z_{P 2}=Z_{C 1}=Z_{C 2}=0$ and $Z_{P 2}=Z_{C 2}=\infty$ and redoing the circuit analysis

$$
\mathrm{TF}_{\text {no plate }}=\frac{\hat{V}}{\hat{p}}=\frac{S}{\phi} \frac{Z_{A}+Z_{T 1}+Z_{T 0}}{Z_{T 0}} .
$$

A complete discussion of this derivation and the expressions for the impedance terms are given in the thesis by Anderson. ${ }^{35}$

The transducers used in the measurements were characterized using electrical impedance measurements to extract average electro-mechanical lumped-element parameters. The geometry and material property values used for the alumina bar are $h=10.2 \mathrm{~mm}$ (measured), $E=391 \mathrm{GPa}$ (measured), $\rho=3956 \mathrm{~kg} / \mathrm{m}^{3}$ (measured), and $\sigma=0.22$ (manufacturer specification). These parameters, along with material properties of the plate and compliant layer, were used to evaluate the expressions for $\mathrm{TF}_{\text {with plate }}$ and $\mathrm{TF}_{\text {no plate }}$.

\section{EXPERIMENT RESULTS}

Experiments were conducted in a water tank that measures $5.3 \times 5.5 \times 7.9 \mathrm{~m}^{3}$ in size. The water tank is kept at a constant temperature and is lined with acoustical absorbing material. A calibrated transmitter or a calibrated receiver can be placed at one end of the tank to transmit/receive sound toward/from a transducer array under test (AUT) at the other end of the tank. A computer controlled turntable can rotate the AUT with fractional degree increments. When the AUT is in a receive mode, up to 64 channels of complex data may be simultaneously acquired by a vector signal analyzer. The advantage of receive mode measurements is that in the postprocessing phase, the array can be arbitrarily steered to any angle by applying appropriate digital delays.

Figure 5 shows a photograph of the array module just before being lowered into the water tank. Two different array configurations were studied, one with a bar and one without. This paper uses the terms plate and bar almost interchangeably. The method is presented in the context of using a plate,

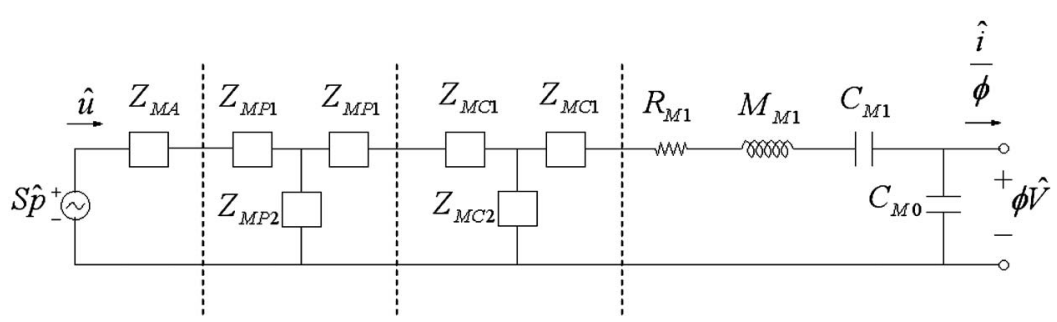

FIG. 4. Equivalent circuit model of an acoustically loaded and plate loaded Tonpilz piezoelectric transducer in a receive condition with waveguide circuits for the plate and compliant layers. 


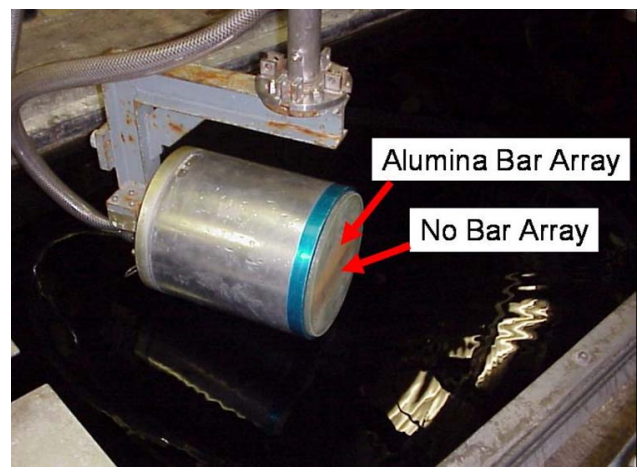

FIG. 5. (Color online) Photograph of the alumina bar array module used in experiments.

but the experiments utilized a bar. Both arrays consisted of transducer elements with nominal resonance frequencies of $25 \mathrm{kHz}$. Both arrays consisted of regularly spaced elements with a center to center spacing $d=30 \mathrm{~mm}$. According to standard line array theory, a grating lobe should be present for all steer angles, $\theta_{0}$, at a frequency of $50 \mathrm{kHz}$. When the array is steered to $\theta_{0}=0^{\circ}$, or broadside, grating lobes should appear at $\pm 90^{\circ}$, according to point source line array theory. However, due to the fact that the elements have finite sizes, with square heads of length $29.5 \mathrm{~mm}$, the directivity of a single element influences the overall directivity pattern according to the first product theorem (or product theorem). ${ }^{3}$

One array was a one-dimensional eight element Tonpilz transducer array that was mounted onto an alumina bar. A 1.6 $\mathrm{mm}$ thick layer of polyurethane separated the transducers from the bar. The alumina bar is a high purity $99.8 \%$ aluminum oxide ceramic (AmAlOx 68 alumina) and is $10.2 \mathrm{~mm}$ thick, $30.5 \mathrm{~m}$ wide, and $244 \mathrm{~mm}$ in length. Plastic shims were placed between neighboring transducers to maintain a consistent spacing between all transducers. The bar was then bonded onto a polyurethane acoustic window of an array module. A second eight-element line array was also bonded directly to the acoustic window without a bar between the array and the window to allow measurements on a similar array without the insertion of the bar. This array module is pictured in Fig. 5. Additional photos of this array module are found in Ref. 35.

\section{A. Directivity pattern measurements}

Directivity pattern measurements were measured with the AUT submersed in the water tank. A calibrated source transducer was placed at the other end of the tank and the AUT was oriented such that the sound energy incident upon the AUT was normal to the array. The calibrated source transducer was also used later in the frequency response measurements. The AUT was placed at a sufficient distance, $r$, away from the source transducer to assume that it was in the far field, $r=r_{\mathrm{FF}}$. The AUT was connected to a turntable that rotated the AUT slowly as the source projected sound energy. The individual element responses, $p_{n}(\theta)$ $=A_{n}(\theta) e^{j \phi_{n}(\theta)}$, were collected at various angles, between $-90^{\circ}$ and $+90^{\circ}$ relative to normal incidence, as the AUT was rotated. In the postprocessing phase, each of the $N$ elements'
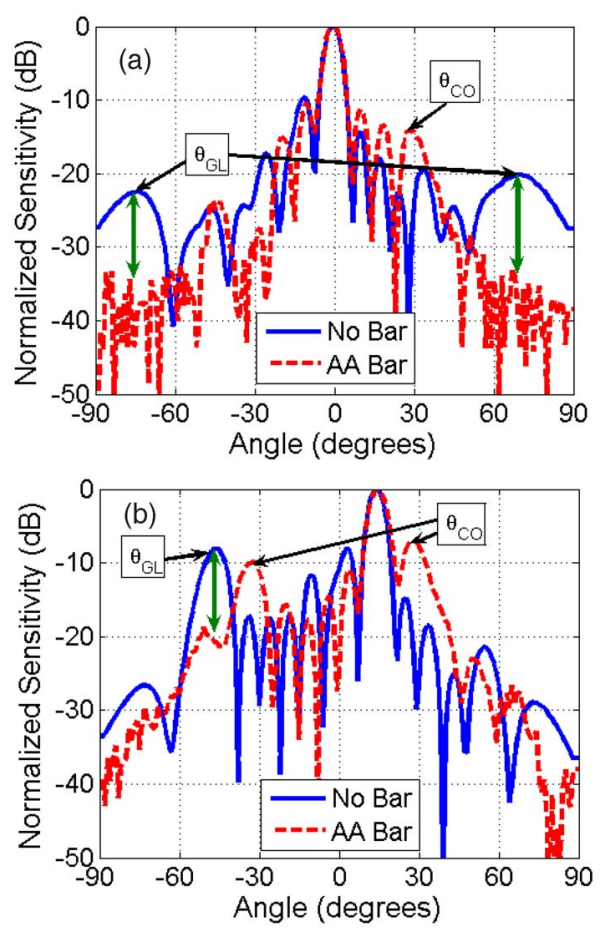

FIG. 6. (Color online) Measured far-field patterns for the alumina (AA) bar array with and without the bar. (a) $50 \mathrm{kHz}$ and $\theta_{0}=0^{\circ}$. (b) $50 \mathrm{kHz}$ and $\theta_{0}$ $=15^{\circ}$.

magnitude, $A_{n}(\theta)$, and phase, $\phi_{n}(\theta)$, could be summed with appropriate steering phases, $i_{n} \omega \tau_{0}$, where $i_{n}$ is the column number and

$$
\tau_{0}=\frac{d \sin \theta_{0}}{c},
$$

where $\theta_{0}$ is the desired steer angle. The combined AUT's directivity pattern for any given steer angle, $p_{\text {AUT }}(\theta)$, is given by

$$
\left.p_{\mathrm{AUT}}(\theta)\right|_{r=r_{\mathrm{FF}}}=\sum_{n=1}^{N} A_{n} e^{j \phi_{n}} e^{j\left(i_{n} \omega \tau_{0}\right)} .
$$

Figure 6 displays the measured directivity patterns for both without-bar and with-bar conditions for the alumina bar array at $50 \mathrm{kHz}$. The patterns displayed in Fig. 6 are normalized with respect to their maxima to allow comparison of relative side lobe and grating lobe levels. The measured patterns for the alumina bar array show distinct peaks at the coincidence angles relative to the no-bar patterns, due to the high degree of sound transmission at the coincidence angles, as predicted in Sec. II. However, at $50 \mathrm{kHz}$ for the alumina bar array, there is a clear reduction in level at the grating lobes. The alumina bar array patterns at $50 \mathrm{kHz}$ reach the measurement system noise floor, approximately between -35 and $-40 \mathrm{~dB}$ in the normalized plots given in Fig. 6, at large angles $>\left| \pm 60^{\circ}\right|$; thus the true reductions in level at these large frequencies cannot be deduced.

Another convenient way to display the directivity pattern data is to plot the directivity patterns for various steer angles as slices in a three-dimensional surface plot. ${ }^{34,35}$ This display format allows one to analyze the effect of the inser- 
(a)

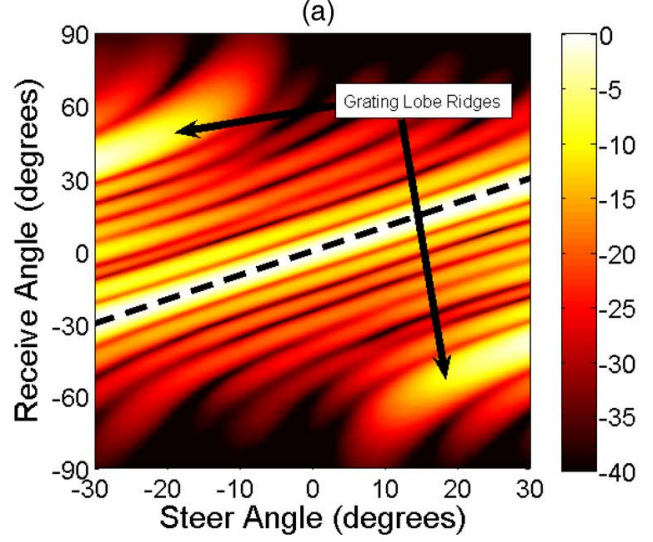

(b)

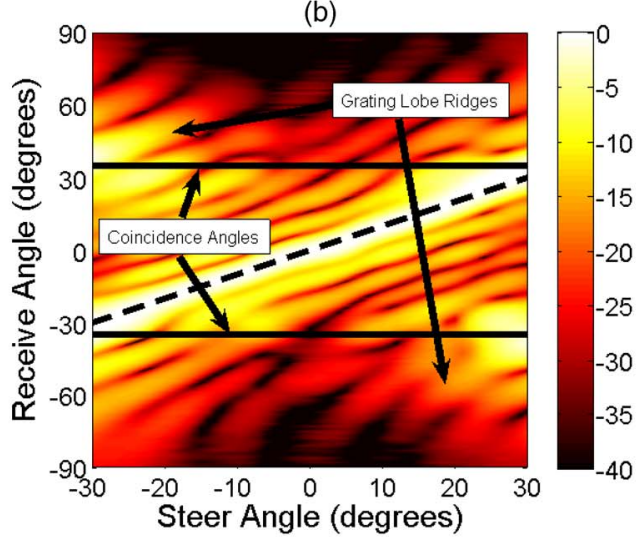

(c)

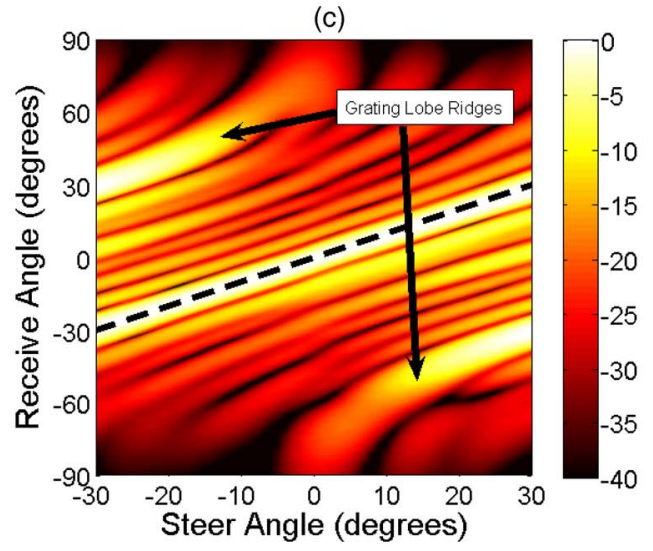

(d)

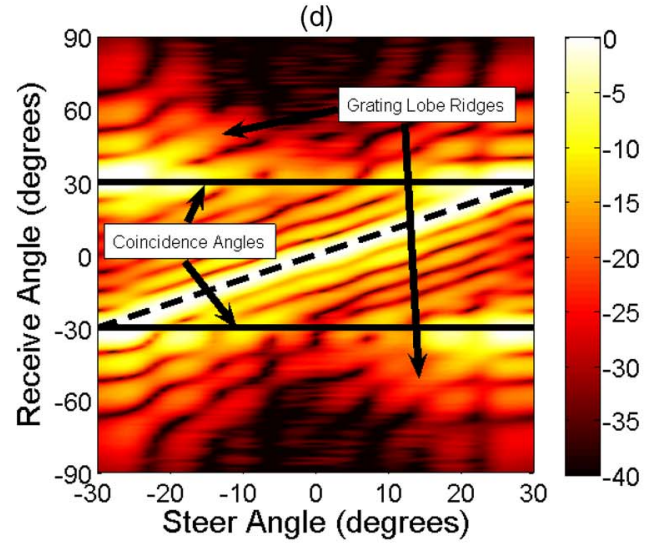

FIG. 7. (Color online) Directivity pattern surface plots for the alumina bar array at $45 \mathrm{kHz}[(\mathrm{a})$ and (b)] and at $50 \mathrm{kHz}[(\mathrm{c})$ and (d)] without the bar [(a) and (c)] and with the bar [(b) and (d)]. The main lobe ridge is identified by the black dashed lines. The grating lobe ridges are also identified. The coincidence angles are identified by the solid black lines.

tion of the bar/plate across many steer angles at the same time. Figure 7 displays directivity pattern surface plots for the alumina bar array, at frequencies of 45 and $50 \mathrm{kHz}$. The grating lobe ridge in both figures is reduced or at least broken up at steer angles less than $\pm 20^{\circ}$. Also, there is an apparent increase in sensitivity at the expected coincidence angles indicated by the black horizontal lines in the figure.

\section{B. Frequency response measurements and simulations}

On-axis frequency response measurements were made with the AUT lowered into one end of the water tank. The total receive response from the AUT was recorded as the source transducer was swept up in frequency. Frequency response measurements were made for the AUT without a bar and then again with a bar and these results are given in Fig. 8. Equations (8) and (12) are evaluated and also plotted in Fig. 8. The presence of the alumina bar introduces a $5-10 \mathrm{~dB}$ transmission loss across the frequency range. Also, the insertion of the alumina bar reduces the $26 \mathrm{kHz}$ resonance in the no-bar frequency response and tends to flatten out the overall response. The on-axis frequency response simulations predict trends found in the measured data, though they do not match the levels correctly. The reason for the model's overestimation of the measured data is unknown. Certain features of the model seem to be shifted down in frequency-note that the knees in the model's prediction at 18 and $34 \mathrm{kHz}$ should match the knees in the measured data at 23 and 42 $\mathrm{kHz}$. The equivalent circuit model includes some low frequency approximations, particularly in the model of the transducers, that explain the discrepancies above $50 \mathrm{kHz}$. While the equivalent circuit modeling shows some promise in modeling frequency response data, further work must be done to improve the modeling, such as using waveguide T-networks to model the transducer's components.

\section{CONCLUSION}

This study represents the first attempt to advantageously utilize a plate to provide angular dependent sound transmis-

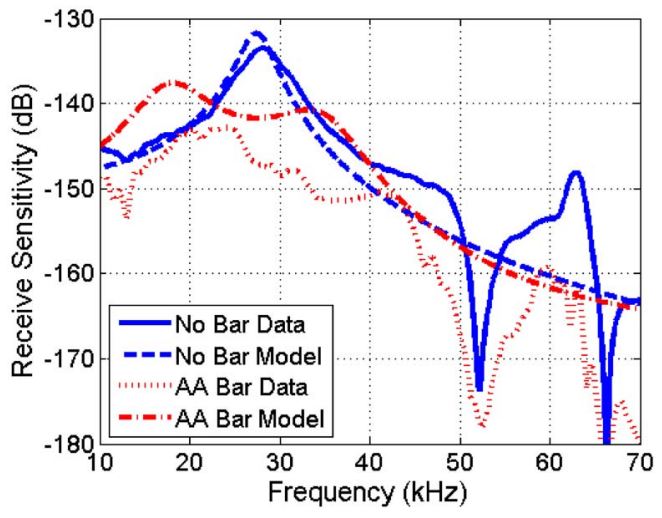

FIG. 8. (Color online) Frequency response comparison plots for the alumina (AA) bar array. The plot displays data and the equivalent circuit model results for the array with and without a bar in place. 
sion filtering of array patterns above the plate's critical frequency (the supercritical frequency region). This study has shown that grating lobes can be reduced due to the insertion of a high stiffness to mass ratio supercritical plate. In addition to reducing grating lobes, the relative stop band transmission loss serves to increase the pattern directivity and suppress levels at large angles where flow noise can be problematic. Use of a plate as an angular filter does restrict steering between the positive and negative coincidence angles. The presence of the plate causes an insertion loss of the main lobe level and a relative increase in transmission in side lobes levels at the coincidence angles; both of these effects depend on the mass per unit area of the plate.

This study has found that while grating lobe levels can be significantly reduced, a practical material has yet to be found for the specific array configuration used. Typical reductions of $10 \mathrm{~dB}$ have been found in the grating lobe level (though the reduction depends on the steer angle of interest), while typical increases of $8 \mathrm{~dB}$ were found in the side lobe level at $\pm 30^{\circ}$. The alumina material tested in this study did not provide optimal results from a practical standpoint, but the physics behind this technique has provided compelling evidence that this technique will succeed in reducing grating lobe levels when a plate is developed which possesses a higher stiffness to mass ratio than currently available materials.

\section{ACKNOWLEDGMENTS}

This research was sponsored by the Office of Naval Research Code No. 333, Dr. David Drumheller. In addition to ONR support for B.E.A., support was also provided by a Penn State University Graduate Research Fellowship, a Penn State College of Engineering Fellowship, an Audio Engineering Society Educational Foundation Award, and Penn State Applied Research Laboratory Exploratory and Foundational Research Program funding. The assistance of Paul Bednarchik, Courtney Burroughs, Bob Dashem, Mark Geleskie, Greg Granville, Bill Thomas, Dave Van Tol, and Mark Wilson of the Penn State Applied Research Laboratory is recognized and appreciated. Facilities located in the Applied Research Laboratory of The Pennsylvania State University were used in this work.

${ }^{1}$ V. M. Albers, Underwater Acoustics Handbook II (The Pennsylvania State University Press, State College, PA, 1965).

${ }^{2}$ D. Stansfield, Underwater Electroacoustic Transducers (Peninsula, Newport Beach, CA, 1991).

${ }^{3}$ D. T. Blackstock, Fundamentals of Physical Acoustics (Wiley, New York, 2000).

${ }^{4}$ O. T. von Ramm, S. W. Smith, and F. L. Thurstone, "Grey scale imaging with complex TGC and transducer arrays," Proc. Soc. Photo-Opt. Instrum. Eng. 70, 266-270 (1975).

${ }^{5}$ S. W. Smith, H. G. Pavey, and O. T. von Ramm, "High speed ultrasound volumetric imaging system-Part 1: Transducer design and beam steering," IEEE Trans. Ultrason. Ferroelectr. Freq. Control 38, 100-108 (1991). ${ }^{6}$ G. R. Lockwood and F. S. Foster, "Optimizing sparse two-dimensional transducer arrays using an effective aperture approach," Proc.-IEEE Ultrason. Symp. 3, 1497-1501 (1994).

${ }^{7}$ E. B. Hutchinson, M. T. Buchanan, and K. Hynynen, "Evaluation of an aperiodic phased array for prostate thermal therapies," Proc.-IEEE Ultrason. Symp. 2, 1601-1604 (1995).

${ }^{8}$ W. J. Zehner, "SONAR array with reduced grating lobes," U.S. Patent $5,414,676(1995)$.
${ }^{9}$ G. R. Lockwood, P. Li, M. O’Donnell, and F. S. Foster, "Optimizing the radiation pattern of sparse periodic linear arrays," IEEE Trans. Ultrason. Ferroelectr. Freq. Control 43, 7-14 (1996).

${ }^{10}$ E. B. Hutchinson and K. Hynyen, "Intracavitary ultrasound phased arrays for noninvasive prostate surgery," IEEE Trans. Ultrason. Ferroelectr. Freq. Control 43, 1032-1042 (1996).

${ }^{11}$ S. A. Goss, L. A. Frizzell, J. T. Kouzmanoff, J. M. Barich, and J. M. Yang, "Sparse random ultrasound phased array for focal surgery," IEEE Trans. Ultrason. Ferroelectr. Freq. Control 43, 1111-1121 (1996).

${ }^{12}$ L. R. Gavrilov, J. W. Hand, P. Abel, and C. A. Cain, "A method of reducing grating lobes associated with an ultrasound linear phased array intended for transrectal thermotherapy," IEEE Trans. Ultrason. Ferroelectr. Freq. Control 44, 1010-1017 (1997).

${ }^{13}$ S. D. Sokka, R. L. King, N. J. McDonald, and K. H. Hynynen, "Design and evaluation of linear intracavitary ultrasound phased array for MRIguided prostate ablative therapies," Proc.-IEEE Ultrason. Symp. 2, 14351438 (1999).

${ }^{14} \mathrm{~A}$. Trucco, "Aperture and element minimization in linear sparse arrays with desired beam patterns," Ultrasonics 38, 161-165 (2000).

${ }^{15}$ G. T. Silva and M. Fatemi, "Linear array transducers for vibroacoustography," Proc.-IEEE Ultrason. Symp. 1, 629-632 (2002).

${ }^{16}$ Y. Liu and Z. Lin, "Active array beamforming using the frequencyresponse masking technique," Circuits and Systems, Proceedings of the 2004 International Symposium on Circuits and Systems, 3, 197-200 (2004).

${ }^{17}$ O. Martinez, G. Godoy, M. A. G. Izquierdo, and L. G. Ullate, "Application of vernier thinning techniques to segmented annular arrays," Ultrasonics 42, 977-982 (2004).

${ }^{18} \mathrm{~S}$. Umemura and C. A. Cain, "Acoustical evaluation of a prototype sectorvortex phased-array applicator," IEEE Trans. Ultrason. Ferroelectr. Freq. Control 39, 32-38 (1992).

${ }^{19}$ O. Martinez, L. G. Ullate, M. A. G. Izquierdo, and T. Sanchez, "Incidence of element distribution on the ultrasonic field of segmented annular arrays," Ultrasonics 38, 176-178 (2000).

${ }^{20}$ O. Martinez, L. G. Ullate, and A. Ibanez, "Comparison of CW patterns from segmented annular arrays and squared arrays," Sens. Actuators, A 85, 33-37 (2000).

${ }^{21}$ K. A. Snook, T. A. Ritter, T. R. Shrout, and K. K. Shung, "Design of a high frequency annular array for medical ultrasound," Proc.-IEEE Ultrason. Symp. 2, 1161-1164 (2001).

${ }^{22}$ O. Martinez, M. Akhnak, L. G. Ullate, and F. Montero de Espinosa, "A small 2D ultrasonic array for NDT applications," NDT \& E Int. 36, 57-63 (2003).

${ }^{23}$ B. P. Hildebrand and G. J. Posakony, "Method and apparatus for energizing an array of acoustic transducers to eliminate grating lobes," U. S. Patent 4,179,683 (1979).

${ }^{24}$ J. R. Talman and G. R. Lockwood, "Theoretical evaluation of a $50 \mathrm{MHz}$ split aperture linear phased array," Proc.-IEEE Ultrason. Symp. 2, 16751678 (1997)

${ }^{25}$ F. J. Pompei and S. C. Wooh, "Phased array element shapes for suppressing grating lobes," J. Acoust. Soc. Am. 111, 2040-2048 (2002).

${ }^{26}$ F. Dupenloup, J. Y. Chapelon, D. Cathignol, and O. Sapozhnikov, "The use of broadband signals to reduce grating lobe effects in HIFU tissue ablation," Proc.-IEEE Ultrason. Symp. 3, 1865-1868 (1994).

${ }^{27}$ J. Y. Chapelon, F. Dupenloup, H. Cohen, and P. Lenz, "Reduction of cavitation using pseudorandom signals," IEEE Trans. Ultrason. Ferroelectr. Freq. Control 43, 623-625 (1996).

${ }^{28}$ F. Dupenloup, J. Y. Chapelon, D. J. Cathignol, and O. A. Sapozhnikov, "Reduction of the grating lobes of annular arrays used in focused ultrasound surgery," IEEE Trans. Ultrason. Ferroelectr. Freq. Control 43, 991998 (1996).

${ }^{29}$ A. Bouakaz, C. T. Lancee, P. J. A. Frinking, and N. de Jong, "Simulations and measurements of nonlinear pressure field generated by linear array transducers," Proc.-IEEE Ultrason. Symp. 2, 1511-1514 (1999).

${ }^{30}$ F. Fahy and P. Gardonio, Sound and Structural Vibration, Radiation, Transmission and Response, 2nd ed. (Academic, Oxford, U.K., 2007).

${ }^{31} \mathrm{~F}$. Hutto, "Wave number-frequency response of free-free Timoshenko beams with multiple sources," MS thesis, The Pennsylvania State University, State College, PA (1986).

${ }^{32} \mathrm{~K}$. Grosh, "An experimental system for measuring the wave numberfrequency response of Timoshenko beams," MS thesis, The Pennsylvania State University, State College, PA (1988).

${ }^{33}$ J. Phillips, "The wave number-frequency filtering characteristics of compliant layers," MS thesis, The Pennsylvania State University, State Col- 
lege, PA (1989).

${ }^{34}$ B. E. Anderson, W. J. Hughes, and S. A. Hambric, "On the steering of sound energy through a supercritical plate by a nearfield transducer array," J. Acoust. Soc. Am. 123, 2613-2619 (2008).

${ }^{35}$ B. E. Anderson, "Grating lobe reduction in transducer arrays through structural filtering of supercritical plates," Ph.D. thesis, The Pennsylvania State University, State College, PA (2006); electronic access available at http://etd.psu.edu (Last viewed June, 2009).

${ }^{36}$ W. P. Mason, Electromechanical Transducers and Wave Filters (Van Nostrand, New York, 1942). 